\title{
Distribution of composite numbers and determination of prime numbers
}

\author{
A. F. Gocgen
}

\begin{abstract}
Integer is either a composite number or a prime number. Therefore, detecting composite numbers is important for solving prime numbers. The study of prime numbers, apart from satisfying human curiosity, can be very important. In this article, the order of composite numbers has been detected. And explained with a simple method and a simple function. And, a method has been developed in which all composite numbers and therefore prime numbers can be determined by using the specified methods, functions and formulas.
\end{abstract}

Keywords: composite number; composite numbers distribution; composite numbers generation; prime number; 


\section{Introduction}

To begin, let's define the composite number and prime number: Composite number is the name given to numbers with more than one prime factor. A prime number is a number that has no factors other than 1 and itself. Also, prime number is infinite. [EW81] An integer is either a composite number or a prime number. Therefore, detecting composite numbers is important for solving prime numbers. The study of prime numbers, apart from satisfying human curiosity, can be very important because, regardless of the choice of counting basis and independent of mathematical notation, prime numbers will have a clear and unambiguous meaning among intelligent beings. Despite the efforts of many curious and smart people, the mystery of prime numbers, specifically their distribution, remains an unsolved problem in mathematics. [Bah21] Also, one of the importance of prime numbers is: Cryptography has gained much more importance today. Asymmetric cryptography as a method is more favored as it is more difficult to break. Asymmetric cryptography is based on prime numbers. The mystery of the prime numbers keeps drawing attention on the subject. [YK17] This article will explain the order of composite numbers. Many formulas will be written to obtain a composite number. And, by detecting composite numbers, prime numbers will be tried to be obtained.

\section{Theorem and Proofs}

\subsection{Yildgenn's Theorem}

This theorem states that composite numbers are in an order.

Let's start by explaining the order simply:

$n=N$

$P_{1}=P_{2}$ :

$P^{2}=$ composite number

$P_{1}^{2}+\left(P_{2} \cdot P_{3}\right)=$ composite number

Example:

Let $n=1 ; P_{1}=P_{2}=2 ; P_{3}=3$ :

$P_{1}^{2}=P_{2}^{2}=2^{2}=4=$ composite number

$P_{1}^{2}+\left(P_{2} \cdot P_{3}\right)=2^{2}+(3 \cdot 2)=10=$ composite number

Let's continue by explaining with function to make it more understandable:

$n=N$

$x_{1}=x_{2}$ :

$f\left(x_{1}\right): P \Longrightarrow N$

$g\left(x_{2}\right): P \Longrightarrow N$

$b\left(x_{3}\right): P \Longrightarrow N$

$f\left(x_{1}\right)=x_{1}^{2}=$ composite number

$b\left(x_{3}\right)=x_{3}^{n}$

$g\left(x_{2}\right)=x_{2} \cdot b$

$(f+g)\left(x_{1}\right)=$ composite number

Example:

Let $n=0 ; x_{1}=x_{2}=7 ; x_{3}=11$ :

$f(7)=7^{2}=49=$ composite number

$b(11)=11^{0}=1$

$g(7)=7 \cdot 1=7$

$(f+g)(7)=56=$ composite number

\subsection{Proofs}

Now it's time to prove the validity of these methods and function. To prove this, let's first remind the definition of composite number. A composite number is the name given to numbers with more than one prime factor. For example, 14 has prime factors of 2 and 7 . Therefore, it is a composite number. Let's focus again on proving the validity of this method and function. 
$n=N$

$P_{1}=P_{2}$ :

The proof that $P^{2}$ forms a composite number is as follows:

Since $\frac{P^{2}}{P}=P$, our result has at least two prime factors, $P$ and $P$.

Therefore, $P^{2}$ forms a composite number.

The proof that $P_{1}{ }^{2}+\left(P_{2} \cdot P_{3}{ }^{n}\right)$ forms a composite number is as follows:

Since $\frac{P_{1}{ }^{2}+\left(P_{2} \cdot P_{3}{ }^{n}\right)}{P_{1}}=P_{1}+\left(P_{2} \cdot P_{3}{ }^{n}\right)$ and $P_{1}=P_{2}$, our results has at least two prime factors, $P_{1}$ and $P_{2}$.

Therefore, $P_{1}{ }^{2}+\left(P_{2} \cdot P_{3}{ }^{n}\right)$ forms a composite number.

$n=N$

$x_{1}=x_{2}$

$f\left(x_{1}\right): P \Longrightarrow N$

$g\left(x_{2}\right): P \Longrightarrow N$

$b\left(x_{3}\right): P \Longrightarrow N$

$f\left(x_{1}\right)=x_{1}^{2}$

$b\left(x_{3}\right)=x_{3}^{n}$

$g\left(x_{2}\right)=x_{2} \cdot b$ :

The proof that $x_{1}^{2}$ forms a composite number is as follows:

Since $\frac{x_{1}^{2}}{x_{1}}=x$, our results has at least two prime factors, $x$ and $x$.

Therefore, $x_{1}^{2}$ forms a composite number.

The proof that $(f+g)\left(x_{1}\right)$ forms a composite number is as follows:

Since $(f+g)\left(x_{1}\right)=x_{1}^{2}+\left(x_{2} \cdot x_{3}^{n}\right), \frac{x_{1}^{2}+x_{2} \cdot x_{3}^{n}}{x_{1}}=x_{1}+\left(x_{2} \cdot x_{3}^{n}\right)$ and $x_{1}=x_{2}$, our results has at least two prime factors, $x_{1}$ and $x_{2}$.

Therefore, $(f+g)\left(x_{1}\right)$ forms a composite number.

\subsection{Other Formulas}

Apart from these methods and function,

$n=N+$

$q\left(x_{1}\right): P \Longrightarrow N+$

$b\left(x_{2}\right): P \Longrightarrow N$

$p(y): N+\Longrightarrow N+$

$x_{1}=x_{2}$

$b\left(x_{2}\right)=x_{2}^{n}$

$$
\begin{gathered}
q\left(x_{1}\right)=\left\{\begin{array}{ll}
x_{1}+b, & \log _{x_{1}} n \geq 2 \\
x_{1}^{2}, & \log _{x_{1}} n<2
\end{array}=y_{1}\right. \\
p(y)=\left\{\begin{array}{ll}
y+b, & \log _{y} n \geq 2 \\
y^{2}, & \log _{y} n<2
\end{array}=y_{2}\right.
\end{gathered}
$$

$y=$ composite number

in its form, a piecewise function can be written.

Example:

Let $n=1 ; x_{1}=x_{2}=7$ :

$$
q(7)=\left\{\begin{array}{ll}
7+b, & \log _{7} 1 \geq 2 \\
7^{2}, & \log _{7} 1<2
\end{array}=y_{1}\right.
$$

$\log _{7} 1 \geq 2=7^{2} \geq 1 \Longrightarrow 7+b$

$b(7)=7^{1}=7$

$7+b=7+7=14=y_{1}=$ composite number

$$
p(14)=\left\{\begin{array}{ll}
14+b, & \log _{1} 14 \geq 2 \\
14^{2}, & \log _{1} 14<2
\end{array}=y_{2}\right.
$$


$\log _{1} 14 \geq 2=14^{2} \geq 1 \Longrightarrow 14+7=21=y_{2}=$ composite number

$$
p(21)=\left\{\begin{array}{ll}
21+b, & \log _{1} 21 \geq 2 \\
21^{2}, & \log _{1} 21<2
\end{array}=y_{3}\right.
$$

$\log _{1} 21 \geq 2=21^{2} \geq 1 \Longrightarrow 21+7=28=y_{2}=$ composite number

To give another example:

Let $n=10 ; x_{1}=x_{2}=3$ :

$$
q(3)=\left\{\begin{array}{ll}
3+b, & \log _{3} 10 \geq 2 \\
3^{2}, & \log _{3} 10<2
\end{array}=y_{1}\right.
$$

$\log _{3} 10<2=9^{2}<10 \Longrightarrow 3^{2}=9=y_{1}=$ composite number

$$
p(9)=\left\{\begin{array}{ll}
9+b, & \log _{9} 10 \geq 2 \\
9^{2}, & \log _{9} 10<2
\end{array}=y_{2}\right.
$$

$\log _{9} 10 \geq 2=9^{2} \geq 81 \Longrightarrow 9+b$

$b(3)=3^{10}=59049$

$9+b=9+59049=59058=y_{2}=$ composite number

The proof of the validity of this piecewise function is:

$n=N+$

$q\left(x_{1}\right): P \Longrightarrow N+$

$b\left(x_{2}\right): P \Longrightarrow N$

$p(y): N+\Longrightarrow N+$

$x_{1}=x_{2}$

$b\left(x_{2}\right)=x_{2}^{n}$

$$
\begin{aligned}
& q\left(x_{1}\right)=\left\{\begin{array}{ll}
x_{1}+b, & \log _{x_{1}} n \geq 2 \\
x_{1}^{2}, & \log _{x_{1}} n<2
\end{array}=y_{1}\right. \\
& p(y)=\left\{\begin{array}{ll}
y+b, & \log _{y} n \geq 2 \\
y^{2}, & \log _{y} n \geq 2
\end{array}=y_{2}:\right.
\end{aligned}
$$

The proof that $x^{2}$ forms a composite number is as follows:

Since $\frac{x^{2}}{x}=x$, our results has at least two prime factors, $x$ and $x$.

Therefore, $x^{2}$ forms a composite number.

The proof that $x_{1}^{2}+x_{2}^{n}$ forms a composite number is as follows:

Since $\frac{x_{1}+x_{2}^{n}}{x_{1}}=x_{2}^{n}$ and $x_{1}=x_{2}$, our results has at least two prime factors, $x_{1}$ and $x_{2}$.

The proof that $y^{2}$ forms a composite number is as follows:

Since $\frac{y^{2}}{y}=y$, our results has at least two prime factors, $y$ and $y$.

Therefore, $y^{2}$ forms a composite number.

The proof that $y+x_{2}^{n}$ forms a composite number is as follows:

Since $\frac{y+x_{2}^{n}}{x_{2}}=y+x_{2}^{n}-1$ and $x_{1}=x_{2}$, our results has at least two prime factors, $x_{1}$ and $x_{2}$.

Therefore, $y+x_{2}^{n}$ forms a composite number.

Also, to get larger composite numbers, a formula can be written like this:

$n_{1}=N+$

$n_{2}=P$

$f\left(x_{1}\right): P \Longrightarrow N$

$g\left(x_{2}\right): P \Longrightarrow N$

$b\left(x_{3}\right): P \Longrightarrow N$

$x_{1}=x_{2}$

$f\left(x_{1}\right)=x_{1}^{2}$ 


$$
\begin{aligned}
& g\left(x_{2}\right)=x_{2} \cdot b \\
& b\left(x_{3}\right)=x_{3}^{n_{1}}
\end{aligned}
$$

$$
\sum_{x_{1}=P}^{n_{2}=P} f+g=k
$$

$k=$ composite number

Example:

Let $n_{1}=1 ; n_{2}=5 ; x_{1}=x_{2}=2 ; x_{3}=3$ :

$$
\sum_{2}^{5} f+g=\left(2^{2}+\left(2 \cdot 3^{1}\right)\right)+\left(3^{2}+\left(2 \cdot 3^{1}\right)\right)+\left(5^{2}+\left(2 \cdot 3^{1}\right)\right)=(4+6)+(9+6)+(25+6)=10+15+31=56=k
$$

$k=$ composite number

The proof that $\sum_{x_{1}=P}^{n_{2}=P} f+g$ forms a composite number is as follows:

$$
\sum_{x_{1}=P}^{n_{2}=P} f+g=\left(x_{1}^{2}+\left(x_{2} \cdot x_{3}^{n_{1}}\right)\right)+\left(x_{4}^{2}+\left(x_{5} \cdot x_{6}^{n_{1}}\right)\right)+\cdots+\left(x_{n_{2}-2}^{2}+\left(x_{n_{2}-1} \cdot x_{n_{2}}^{n_{1}}\right)\right)
$$

Since $\frac{\sum_{x_{1}=P}^{n_{2}=P} f+g}{x_{1}}=\frac{\left(x_{1}^{2}+\left(x_{2} \cdot x_{3}^{n_{1}}\right)\right)+\left(x_{4}^{2}+\left(x_{5} \cdot x_{6}^{n_{1}}\right)\right)+\cdots+\left(x_{n_{2}-2}^{2}+\left(x_{n_{2}-1} \cdot x_{n_{2}}^{n_{1}}\right)\right)}{x_{1}}=\left(x_{1}+\left(x_{2} \cdot x_{3}^{n_{1}}\right)\right)+\left(x_{4}^{2}+\left(x_{5}\right.\right.$. $\left.\left.x_{6}^{n_{1}}\right)\right)+\cdots+\left(x_{n_{2}-2}^{2}+\left(x_{n_{2}-1} \cdot x_{n_{2}}^{n_{1}}\right)\right)$ and $x_{1}=x_{2}$, our results has at least two prime factors, $x_{1}$ and $x_{2}$.

Therefore, $\sum_{x_{1}=P}^{n_{2}=P} f+g$ forms a composite number.

To get even larger composite numbers, a formula can be written like this:

$n_{1}=N+$

$n_{2}=P$

$f\left(x_{1}\right): P \Longrightarrow N$

$g\left(x_{2}\right): P \Longrightarrow N$

$b\left(x_{3}\right): P \Longrightarrow N$

$x_{1}=x_{2}$

$f\left(x_{1}\right)=x_{1}^{2}$

$g\left(x_{2}\right)=x_{2} \cdot b$

$b\left(x_{3}\right)=x_{3}^{n_{1}}$

$$
\prod_{x_{1}=P}^{n_{2}=P} f+g=l
$$

$l=$ composite number

Example:

Let $n_{1}=1 ; n_{2}=5 ; x_{1}=x_{2}=2 ; x_{3}=3$ :

$$
\prod_{2}^{5} f+g=\left(2^{2}+\left(2 \cdot 3^{1}\right)\right) \cdot\left(3^{2}+\left(2 \cdot 3^{1}\right)\right) \cdot\left(5^{2}+\left(2 \cdot 3^{1}\right)\right)=(4+6) \cdot(9+6) \cdot(25+6)=10 \cdot 15 \cdot 31=4650=l
$$

$l=$ composite number

The proof that $\prod_{x_{1}=P}^{n_{2}=P} f+g$ forms a composite number is as follows:

$$
\prod_{x_{1}=P}^{n_{2}=P} f+g=\left(x_{1}^{2}+\left(x_{2} \cdot x_{3}^{n_{1}}\right)\right) \cdot\left(x_{4}^{2}+\left(x_{5} \cdot x_{6}^{n_{1}}\right)\right) \cdots\left(x_{n_{2}-2}^{2}+\left(x_{n_{2}-1} \cdot x_{n_{2}}^{n_{1}}\right)\right)
$$


Since $\frac{\prod_{x_{1}=P}^{n_{2}=P} f+g}{x_{1}}=\frac{\left(x_{1}^{2}+\left(x_{2} \cdot x_{3}^{n_{1}}\right)\right) \cdot\left(x_{4}^{2}+\left(x_{5} \cdot x_{6}^{n_{1}}\right)\right) \cdots \cdot\left(x_{n_{2}-2}^{2}+\left(x_{n_{2}-1} \cdot x_{n_{2}}^{n_{1}}\right)\right)}{x_{1}}=\left(x_{1}+\left(x_{2} \cdot x_{3}^{n_{1}}\right)\right) \cdot\left(x_{4}^{2}+\left(x_{5}\right.\right.$. $\left.\left.x_{6}^{n_{1}}\right)\right) \cdots\left(x_{n_{2}-2}^{2}+\left(x_{n_{2}-1} \cdot x_{n_{2}}^{n_{1}}\right)\right)$ and $x_{1}=x_{2}$, our result has at least two prime factors, $x_{1}$ and $x_{2}$.

Therefore, $\prod_{x_{1}=P}^{n_{2}=P} f+g$ forms a composite number.

\section{$3 \quad$ Inferences}

Regarding the Yildgenn Theorem, the following inferences can be made from the methods and functions stated so far:

As mentioned earlier, an integer is either a composite number or a prime number.

This means that detecting all composite numbers means detecting all prime numbers.

As stated in the Yildgenn Theorem:

$n=N$

$P_{1}=P_{2}$ :

$P^{2}=z_{1}$

$P_{1}^{2}+\left(P_{2} \cdot P_{3}\right)=z_{2}$

$z=$ composite number

Let's edit this method so that it works better for us: $P_{1}$, changed to $x_{1}=$ odd prime (Except for 2 , since it is not an even prime number, it has been changed to a single prime to avoid computational congestion.).

$P_{2}$, changed to $x_{2}=$ odd prime (Except for 2 , since it is not an even prime number, it has been changed to a single prime to avoid computational congestion.).

$P_{3}$, changed to 2 (Since we want to detect all prime numbers, we aim to detect all odd (the reason it is specified as odd, as mentioned before, there is no even prime number other than 2 , that is to prevent processing density) composite numbers. To detect all odd composite numbers, and therefore all prime numbers, we replace it with the smallest prime number, (composite number: the name given to numbers with more than one prime factor) so 2.):

$$
\begin{aligned}
& n=N \\
& x_{1}=\text { odd prime } \\
& x_{2}=\text { odd prime } \\
& x_{1}=x_{2} \\
& x_{1}^{2}=z_{1} \\
& z_{1}+\left(x_{2} \cdot 2\right)=z_{2} \\
& z_{2}+\left(x_{2} \cdot 2\right)=z_{3} \\
& \ldots \\
& z=\text { odd composite number }
\end{aligned}
$$

If $x$ is given all odd prime values linearly from the smallest odd prime to the largest odd prime, the resulting odd numbers between any two z-values or between 0-smallest z-values and not equal to any z-values are prime.

Example:

Suppose the only, only prime number we know is 3 :

$3^{2}=9=z_{1}$

$9+(3 \cdot 2)=15=z_{2}$

We know that odd numbers between any two z-values and not equal to any z-values are prime. In this case, odd numbers between $z_{1}$ and $z_{2}$ that do not equal any $z$ value are prime.

The odd numbers that do not equal any $\mathrm{z}$ value between $z_{1}$ and $z_{2}$ are 11 and 13 .

So 11 and 13 are prime.

Also, all odd numbers with the between 0 -smallest z-value are also prime. Because there cannot be a $z$-value smaller than the smallest z-value. In this case, it is also impossible for any value less than the smallest $\mathrm{z}$ value to be equal to any $\mathrm{z}$ value.

So, 5 and 7 are also prime.

$15+(3 \cdot 2)=21=z_{3}$

The numbers between $z_{2}$ and $z_{3}$ that do not equal any $z$ value are: 17,19 .

We learned that the numbers 17 and 19 are also prime. 
$5^{2}=25=z_{4}$

The number between $z_{3}$ and $z_{4}$ that is not equal to any z-value is 23 . So 23 is prime.

$21+(3 \cdot 2)=27=z_{5}$

There is no number between $z_{4}$ and $z_{5}$ that does not equal any $z$ value.

$27+(3 \cdot 2)=33=z_{6}$

The numbers between $z_{5}$ and $z_{6}$ that do not equal any z value are 29 and 31 . So these two numbers are prime.

...

The example can be continued in this way.

\section{Results}

The order of composite numbers has been detected. And explained with a simple method and a simple function. The validity of the method and function has been proven. Using this simple method and function, other formulas were written in which composite numbers could be determined. The validity of these formulas was then proven. Finally, a method has been developed in which all composite numbers and therefore prime numbers can be determined by using the specified methods, functions and formulas.

\section{References}

[Bah21] D. Bahrami. An observation on distribution of prime numbers. Preprints, (2021060416 (doi: 10.20944/preprints202106.0416.v1)), 2021.

[EW81] Euclid and J. Williamson. The elements of euclid. with dissertations. 1781.

[YK17] Tarık Yerlikaya and Onur Kara. Prime number test algorithms used in cryptology. Trakya University Journal of Engineering Sciences, 18(1):85-94, 2017. 